International Journal of Neural Systems, Vol. 28, No. 0 (2018) 1850006 (17)pages)

(C) The Author(s)

DOI: $10.1142 / \mathrm{S} 0129065718500065$

\title{
Influence of Cholecystokinin-8 on Compound Nerve Action Potentials from Ventral Gastric Vagus in Rats
}

\author{
Khalid B. Mirza*, ${ }^{*}$ Andrea Alenda*,§, Amir Eftekhar*, Nir Grossman*, \\ Konstantin Nikolic*, ${ }^{*}$, Stephen R. Bloom ${ }^{\dagger}$ and Christofer Toumazou*,\| \\ *Institute of Biomedical Engineering \\ Department of Electrical and Electronic Engineering \\ Imperial College London, SW' $2 A Z, U K$ \\ ${ }^{\dagger}$ Division of Diabetes, Endocrinology and Metabolism \\ Section of Endocrinology and Investigative Medicine \\ Imperial College London, W12 0NN, UK \\ †k.mirza@imperial.ac.uk \\ §a.alenda@imperial.ac.uk \\ 『k.nikolic@imperial.ac.uk \\ "I.toumazou@imperial.ac.uk
}

Accepted 4 February 2018

Published Online 9 April 2018

\begin{abstract}
Objective: Vagus Nerve Stimulation (VNS) has shown great promise as a potential therapy for a number of conditions, such as epilepsy, depression and for Neurometabolic Therapies, especially for treating obesity. The objective of this study was to characterize the left ventral subdiaphragmatic gastric trunk of vagus nerve (SubDiaGVN) and to analyze the influence of intravenous injection of gut hormone cholecystokinin octapeptide (CCK-8) on compound nerve action potential (CNAP) observed on the same branch, with the aim of understanding the impact of hormones on VNS and incorporating the methods and results into closed loop implant design. Methods: The cervical region of the left vagus nerve (CerVN) of male Wistar rats was stimulated with electric current and the elicited CNAPs were recorded on the SubDiaGVN under four different conditions: Control (no injection), Saline, CCK1 (100 pmol/ kg) and $C C K 2(1000 \mathrm{pmol} / \mathrm{kg})$ injections. Results: We identified the presence of $\mathrm{A} \delta, \mathrm{B}, \mathrm{C} 1, \mathrm{C} 2$, C3 and $\mathrm{C} 4$ fibers with their respective velocity ranges. Intravenous administration of CCK in vivo results in selective, statistically significant reduction of CNAP components originating from A and B fibers, but with no discernible effect on the $\mathrm{C}$ fibers in $n=7$ animals. The affected CNAP components exhibit statistically significant $\left(p_{\text {Saline-CCK1 }}=0.02\right.$ and $\left.p_{\text {Saline-CCK2 }}=0.007\right)$ higher normalized stimulation thresholds. Conclusion: This approach of characterizing the vagus nerve can be used in closed loop systems to determine when to initiate VNS and also to tune the stimulation dose, which is patientspecific and changes over time.
\end{abstract}

Keywords: Compound nerve action potentials; subdiaphragmatic vagus nerve; vagus nerve stimulation; closed loop system.

\section{Introduction}

The vagus nerve is the longest cranial nerve originating directly from the brain and branching downwards into the body. It emerges from the medulla oblongata, traveling along the two lateral sides of the body, innervating organs in thoracic and abdominal regions! 1 The vagus nerve acts as a conduit for information exchange in the form of neural

\footnotetext{
『 Corresponding author.

This is an Open Access article published by World Scientific Publishing Company. It is distributed under the terms of the Creative Commons Attribution 4.0 (CC-BY) License. Further distribution of this work is permitted, provided the original work is properly cited.
} 
signals between the brain and the organs it innervates. Neuromodulation ${ }^{2}$ is an emerging therapeutic treatment modality for a number of intractable diseases. The multifunctional and highly distributed nature of the vagus nerve has led to vagus nerve stimulation (VNS) being explored as a neuromodulatory therapeutical target for a number of applications ranging from pharmocoresistant eplilepsy, depression to obesity. ${ }^{\sqrt[3]{6}}$ An emerging neuromodulatory obesity management technique is $\mathrm{VNS}^{7}$

Vagal afferents in the gastrointestinal (GI) tract play an important role in determining meal sizes, with mechanosensitive vagal afferents responding to distension and chemosensitive vagal afferents responding to nutrient type and quantity $[$ VNS has shown traction in reducing weight with minimal invasiveness and surgical impact on the body. Data from ReCharge (2011-2013 $\sqrt{5}^{5}$ and EMPOWER $(2011)^{9}$ studies have demonstrated the clinical feasibility of using VNS as an obesity management technique and have also supported commercially available solutions such as the VBLOC Maestro ${ }^{\mathrm{TM}}$ system, a VNS implant for obesity management by EnteroMedics $^{\mathrm{TM}}$. The implant, recently approved by FDA, can be surgically placed with the help of laproscopic techniques, hence, it is surgically less invasive compared to gastric bypass surgery (GBS) 10

Majority of VNS applications involve open loop stimulation. However, studies have shown that open loop VNS, where stimulation parameters are preprogrammed and not affected by patient's physiological condition, leads to modest weight loss only $\underline{[1619}$ Modest success rates have impeded the adoption of VNS as a low risk, low cost replacement to GBS

Within a closed loop neuromodulation paradigm, the initiation of stimulation and the dosage of stimulation are controlled by physiological events. ${ }^{[11} \mathrm{In}$ the context of weight loss, an implant with closed loop capabilities is Intrapace abiliti ${ }^{\mathrm{TM}_{\mathrm{M}}}$ which initiates Gastric Electrical Stimulation (GES) in response to transit of food in the stomach, such as during meal intake ${ }^{12}$ Another implant, the Diamond ${ }^{\mathrm{TM}}$ (Metacure) system, stimulates the gastric antrum when food is ingested in the stomach. It has also shown greater weight loss capability than open loop implants. ${ }^{13}$ Both implants do not stimulate the vagus nerve directly, however, the stimulation might be affecting vagal afferents in the stomach wall.
Recent studies on $\mathrm{VBLOC}^{\mathrm{TM}}$, the only commercially available Gastric-VNS implant, indicated that a possible mechanism with which VNS using VBLOC $^{\mathrm{TM}}$ leads to weight loss is by blocking efferent fibers carrying vagal signals to the gut 14 In this paper, an effort has been made to determine if gut hormone signaling through vagal afferents can be used within the closed loop VNS modality. Gut hormones such as cholecystokinin (CCK), glucagon, glucagon-like peptide-1 (GLP-1) are considered to be essential in appetite regulation. CCK plays an important role in the complex negative feedback to the brain to initiate meal termination. ${ }^{[5}$ Previous work ${ }^{16117}$ has suggested that CCK leads to appetite suppressive effect. It activates CCKA receptors which are expressed on afferent terminals of the vagus nerve present in the gut 10 CCK introduced exogenously also leads to satiation, reduced motility, release of gastric and pancreatic enzymes involved in digestion.10

By detecting the release of gut hormones through compound nerve action potentials (CNAPs) and initiating VNS, there is a possibility to automate the initiation of stimulation. Also, analysis of CNAPs and interrogating the state of the nerve will enable better control of stimulation dosage ${ }^{18}$ The start of high frequency VNS, such as $\mathrm{VBLOC}^{\mathrm{TM}}$ in relation to release of gut hormones may have the potential of amplifying the satiety-related impact of gut hormones.

It has also been demonstrated that the satiating effect of CCK-8 is reduced after abdominal vagotomy leading to the conclusion that vagal afferents play an important role in producing the satiation response in conjunction with CCK ${ }^{19}$ As the subdiaphragmatic gastric trunk of vagus nerve (SubDiaGVN) is responsible for carrying afferent signals from the gut to the brain and efferent signals from the brain to the gut, in this paper, we explore the possibilities of interrogating the SubDiaGVN branch, by electrical stimulation at a distant location and recording locally, to determine the presence of different fiber types and to correlate the CNAP response with the physiological condition of the targeted organ (e.g. stomach), and develop a bio-marker for providing feedback to the VNS device.

The eventual goal is to identify the effect of CCK released during meal ingestion on vagus and initiate 
VNS to affect appetite early on. Hence, we characterize the SubDiaGVN branch of the vagus nerve, by determining the different fiber types present, respective velocity ranges for each fiber type and its relative stimulation threshold based on CNAPs observed in response to electrical neural stimulation under four physiological conditions: Control, Saline, CCK1 and $C C K 2$. Prechtl et al..$^{20}$ have shown that, anatomically in the abdominal vagus of rats, $28 \%$ of the fibers are motor fibers (A and B fibers) and $72 \%$ fibers are sensory fibers (C fibers) $! 21]$

Due to the differences in velocities for different fiber types, CNAPs can be used as a method to determine activity originating from different fiber types and their respective stimulation thresholds which can be used to determine the optimal stimulation dose. Nerve conduction velocities are also used to assess nerve damage due to demylenation or axonal degeneration.21 This is followed by an analysis of the impact of different physiological conditions (Control i.e. no injection, Saline and CCK injection) on CNAP metrics (such as amplitude, activation threshold, etc.).

CNAPs were chosen because of their larger signal amplitude $(\geq 5 \mu \mathrm{V})$, compared to measuring sub- $\mu \mathrm{V}$ neural mass activity changes; a significant challenge to detect with even the current state-of-the-art in neural recording technology. Due to the differences in conduction velocities for different fiber types, CNAPs can be used to determine activity originating from different fiber types and their respective stimulation thresholds. This can be used to adjust the optimal stimulation dose. In general, nerve conduction velocities are also used to assess nerve damage due to demyelination or axonal degeneration. In summary, Sec. 2 describes the in vivo experimental setup used. Section 3 gives a detailed description of the analytical methods used to analyze the experimental data. The results are presented in Sec. 团followed by a discussion in Sec. 5 .

\section{Methods}

All surgical and experimental protocols were performed in accordance with the Animal (Scientific Procedures) Act 1986 and following local ethical approval.

\subsection{In vivo experimental methods}

\subsubsection{Animals}

Male Wistar Rats, obtained from Charles River, were used in all experiments. The animals $(n=7)$ were permitted ad libitum access to food and water and a $12 \mathrm{~h}$ light:dark cycle was maintained over them.

\subsubsection{Cervical region of the left vagus nerve (CerVN) and SubDiaGVN preparation}

Rats were anesthetized with isoflourane followed by urethane $\left(20 \mathrm{mg} \cdot \mathrm{kg}^{-1}\right)$ which was infused at a slow rate over $25 \mathrm{~min}$. Urethane was chosen as evidence which appears to suggest that it does not affect peripheral nerve activity ${ }^{22}$ Urethane also gives a very stable anesthetic state throughout the procedure compared to other anesthetics such as pentobarbital or ketamine/xylacine and has been used in previous CCK- 8 studies. ${ }^{19}$ In order to assess full anesthesia state, the absence of forepaw pinch reflex was verified. During the procedure, heart rate and oxygen saturation were monitored through a pulse oximeter (Physiosuite, Kent Scientfic). The body temperature was maintained at $37 \pm 1^{\circ} \mathrm{C}$ by using a heat pad and measured with a rectal thermistor (Physiosuite, Kent Scientific).

After full anesthetization, a midline incision was made to expose the CerVN branch for stimulation. In order to record from the SubDiaGVN, a midline incision was made in abdomen. The connective tissue around the liver was gently removed. A large cotton bud was used to gently displace the liver. The liver was not bruised in the procedure and was easily put back in place. The esophagus and the anterior SubDiaGVN, present nearby between the diaphragm and top of the stomach, were exposed. A thread was used to lift the nerve, and a recording electrode was placed under the SubDiaGVN, just below the hepatic branch, as the subdiagphragmatic ventral trunk just enters the stomach (for more details, see Fig. A.1).

A pseudo-tripolar cuff electrode was placed under the left cervical vagus nerve for stimulation. A pair of similar bipolar electrodes were placed, in the cervical cavity and subdiaphragmatic cavity on the nearby tissue to capture any in-band interferences due to movement or electromyographic (EMG) activity, which share the same bandwidth as CNAPs. 
Because interferences are common to vagus and reference channels and CNAPs are present only on the vagus recording channel, it is possible to separate CNAPs from interferences.

The approximate distance between stimulation and recording electrodes was $8 \pm 0.5 \mathrm{~cm}$. The cavities were filled with paraffin oil as an insulation medium as described in previous papers. $23 \sqrt{26}$

\subsubsection{Electrodes}

The bipolar recording electrodes were fabricated inhouse by utilizing temper annealed $125 \mu \mathrm{m}$ platinum (Pt) wires (Advent Research Materials). The $\mathrm{Pt}$ wires were cleaned by heating them to very high temperature with a flame torch. After cleaning, the Pt wire was soldered to a stainless steel wire. Multiple layers of heat shrink were applied to the junction of $\mathrm{Pt}$ and steel wire. In order to seal the junction, the open ends of the heat shrink were sealed using Araldite glue. A similar procedure was followed for pseudo-tripolar stimulation electrodes.

\subsubsection{Physiological conditions}

The studies involving CCK comprise of recording CNAPs under four different conditions with regards to intravenous injections: Control is the condition where no intravenous injection takes place, Saline is the condition after injection of saline solution. Two different doses of CCK-8 (Tocris Biosciences, UK) were injected: a low dose of $100 \mathrm{pM}$ per $\mathrm{kg}$ (condition $C C K 1$ ) and a high dose of $1000 \mathrm{pM}$ per $\mathrm{kg}$ (condition $C C K 2$ ). Prior to injection, CCK-8 was dissolved in saline and the required amount was drawn into $250 \mu \mathrm{L}$ syringe (Hamilton). The CCK dose was delivered through a tail vein cannula followed by a flush of $250 \mu \mathrm{L}$ saline.

\subsection{Recording and stimulation setup}

Figure 1 shows an overview of the experimental setup.

Vagal nerve CNAPs were recorded using the Intan RHD 2132 Electro-physiological recording chip. For data acquisition, RHD 2000 Amplifier Evaluation system and software were utilized. The RHD 2132 has 16 bipolar channels out of which 3 channels were utilized. One of the channels was used to record CNAPs on the vagus nerve and two other channels

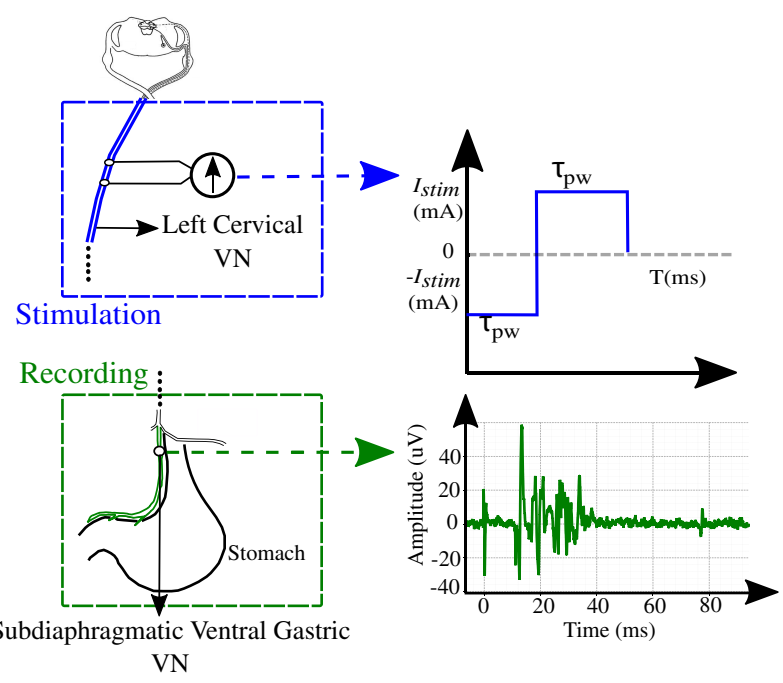

Fig. 1. The stimulation and recording setup positioned at the left cervical and subdiaphragmatic branches of the vagus nerve, respectively (left). A typical biphasic stimulus (pulsewidth $\tau_{\mathrm{pw}}$ and amplitude $I_{\mathrm{stim}}$ ) and recorded CNAP (right) are shown.

were used as reference channels in the cervical and subdiaphragmatic cavities ${ }^{27}$ The cervical reference channel ensured that stimulation of CerVN did not lead to stimulation of nearby neck muscles, hence loss of stimulation strength. The stimulation artifact observed in the subdiaphragmatic reference channel and the vagus recording channel helped in aligning waveforms in time (Fig. A.2).

The stimulation and recording equipment were connected to a single grounding point, as in star grounding configuration. The animal was also grounded to this single ground point by placing a silver-silver chloride $(\mathrm{Ag} / \mathrm{AgCl})$ electrode under the skin of the animal. On the ventral side, a differential bipolar recording configuration was implemented to ensure better rejection of common mode signals. A digital bandpass filter $(200 \mathrm{~Hz}-7.2 \mathrm{kHz})$ was also activated using the RHD2000 Amplifier Evaluation software to match the bandwidth of CNAPs and reduce noise in the neural recording. In order to remove line interference, a $50 \mathrm{~Hz}$ digital notch filter was activated using RHD 2000 Amplifier Evaluation software. A current stimulation setup was designed by using Keithley 6221 current source (Tektronix). The programmable stimulation protocols were downloaded into the Keithley 6221 through a MATLAB script and GPIB I/O protocol. 


\subsubsection{Stimulation protocol}

During each injection condition, the same CerVN stimulation protocol was followed in which biphasic current pulses were used. The pulsewidths were $\tau_{\mathrm{pw}}=0.1,0.2,0.5,1$ and $5 \mathrm{~ms}$. For every pulsewidth, the stimulation current $I_{\text {stim }}$ was cycled through 16 different values. For pulsewidths $0.1 \mathrm{~ms}$ and $0.2 \mathrm{~ms}$, the stimulation current ranges from $0.2 \mathrm{~mA}$ up to $3 \mathrm{~mA}: I_{\text {stim }}=0.2,0.5,0.8,1-1.8 \mathrm{~mA}$ $\left(\Delta I_{\text {stim }}=0.1 \mathrm{~mA}\right), 1.8-3 \mathrm{~mA}\left(\Delta I_{\text {stim }}=0.2 \mathrm{~mA}\right)$. For pulsewidths $0.5,1$ and $5 \mathrm{~ms}$, the stimulation current ranges from $0.1 \mathrm{~mA}$ up to $2 \mathrm{~mA} I_{\text {stim }}=0.1-1.2 \mathrm{~mA}$ $\left(\Delta I_{\text {stim }}=0.1\right), 1.2-2 \mathrm{~mA}\left(\Delta I_{\text {stim }}=0.2\right)$. The stimulation frequency was $f_{\text {stim }}=2 \mathrm{~Hz}$, each set of stimulation setups was repeated 10 times, and the break between stimulation sessions was $10 \mathrm{~min}$.

\section{Analytical Methods}

The fiber type classification was based on the CNAP conduction velocity and fiber grouping was done into distinct velocity bands, $\stackrel{28}{28}$ see Fig. [2.

The recorded CNAP waveforms are preprocessed to ensure that no in-band interferences are present and to reduce noise in the waveform (Fig. A.3). After preprocessing, a threshold is applied to the waveform and positive/negative peaks above/below the threshold are detected. The peaks representing fiber components with distinct velocities were used to construct a velocity profile of the nerve.

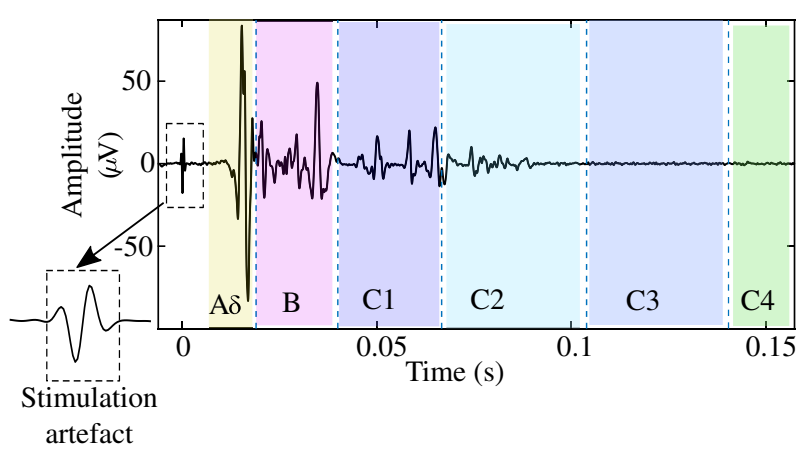

Fig. 2. CNAP recorded on the SubDiaGVN showing the stimulation artifact and the different fiber regions, elicited at stimulation current $1.6 \mathrm{~mA}$, pulsewidth $0.2 \mathrm{~ms}$, and stimulation frequency $2 \mathrm{~Hz}$. The regions are based on the conduction velocity calculations with the stimulation and recording sites separated by $8 \mathrm{~cm}$.

\subsection{Compound nerve action potentials}

A CNAP waveform is the resultant sum of all individual fiber action potentials present in the nerve. $\underline{29}$ By ensuring that there is enough separation between stimulation and recording electrodes, as mentioned in Sec. 2, we were able to obtain better resolution of CNAP components based on their latencies. An example of our recordings is shown in Fig. 3 ,

\subsection{Preprocessing}

Preprocessing involved comparison of vagal recording channel with the cervical and subdiaphragmatic reference recording channels to differentiate between
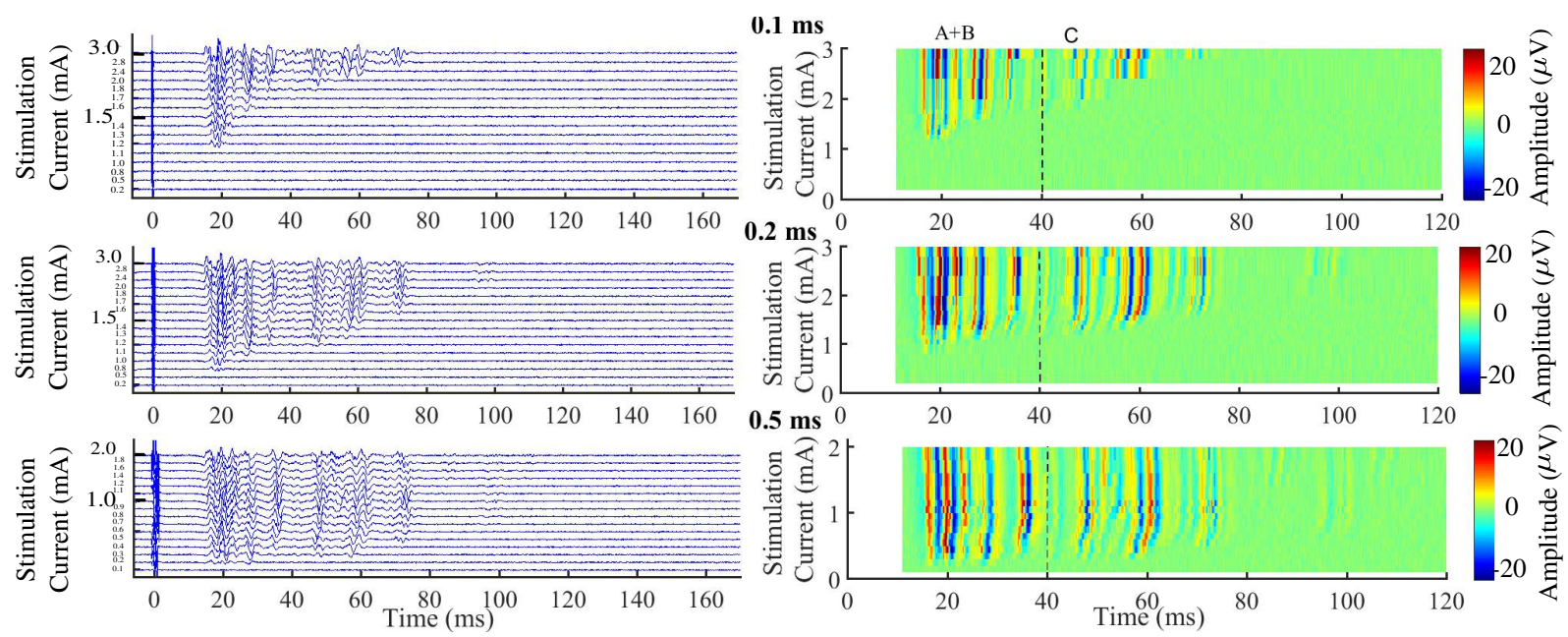

Fig. 3. The CNAPs elicited for three different pulsewidths and 16 different stimulation currents $\left(\tau_{\mathrm{pw}}=0.1 \mathrm{~ms}, 0.2 \mathrm{~ms}\right.$, $I_{\mathrm{stim}}=1-3 \mathrm{~mA}$ and $\tau_{\mathrm{pw}}=0.5 \mathrm{~ms}, I_{\text {stim }}=1-2 \mathrm{~mA}$, see Stimulation Protocol for details): (left) recorded traces, offset by $0.2 \mathrm{~mA}$, and (right) a color map representation of the CNAPs. 
CNAPs and interferences, see (Fig. A.3). The elicited CNAP waveform is aligned with the reference channels using the stimulation artifact (see Fig. A.2). Correlation is then used to determine any similar components present, which will be ignored during further analysis.

The CNAP waveforms due to 10 repeated stimuli are averaged to reduce the noise in recordings and also to reduce the impact of in-band artifacts, if present. After averaging, the peaks are detected above the noise level of the recording system at a threshold of $V_{\mathrm{th}}=5 \mu \mathrm{V}$, Fig. 固 $\mathrm{a}$ ).

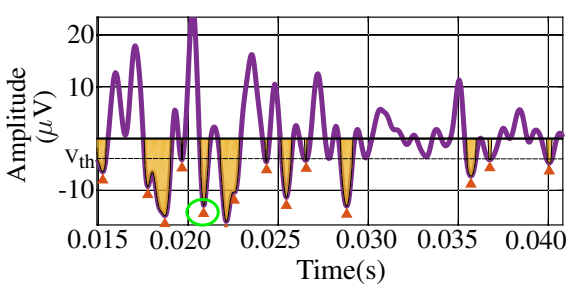

(a)

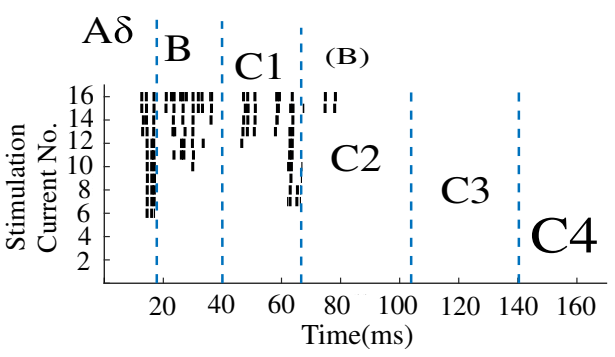

(b)

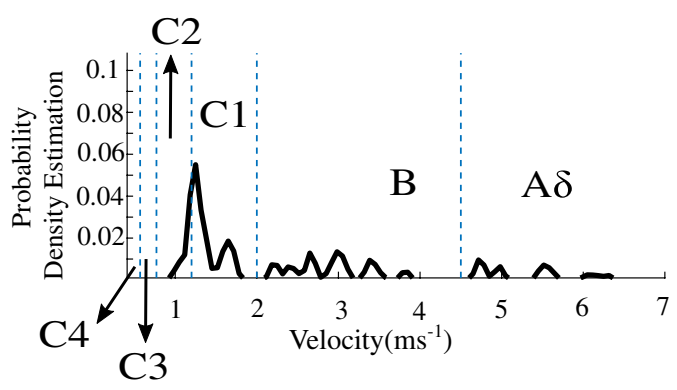

(c)

Fig. 4. (a) CNAP waveforms in which negative/ positive peaks are detected below/above a certain amplitude threshold and the area under the curve is determined. (b) The peak locations are used to construct a raster plot showing different fiber events. (c) Fiber events are used to construct a velocity profile for the fiber.

\subsection{Velocity profile}

The presence of a peak indicates the activation of a nerve fiber or a population of nerve fibers with specific velocity. The distance between the stimulationrecording electrode and temporal location of the peaks are used to calculate the velocity of the CNAP component. The occurrence of a certain fiber component, known as a fiber event, was noted in the form of a raster plot, Fig. 4 (b). After combining the number of times the events for a particular fiber type have occurred, a velocity profile of the nerve is constructed, such that the velocity profile represents the probability density function of different fiber types represented by different velocities, Fig. 4(c). The fiber event probability density (FEvPD) is calculated using the Parzen window density estimation:

$$
P(v)=\frac{1}{n} \frac{1}{\sigma} \sum K\left(\frac{v-v_{i}}{\sigma}\right),
$$

where $n$ is the total sum of unique fiber events across all 16 stimulation currents (for a given pulsewidth), $\sigma$ is the bandwidth parameter and $v_{i}$ is a fiber event. $K(v)$ is the kernel function, in our case it is Gaussian. The bandwidth parameter $\sigma$ corresponds to the width of the kernel $(\sigma=0.05$ was chosen for all experiments). It determines the smoothness of the PDF curve.

The velocity profile of a fiber gives an indication of the likelihood of excitation and spread in velocity for a range of currents at a specific pulsewidth. Further velocity analysis included determining the fiber velocity with the highest FEvPD and whether this changed across pulsewidths and conditions within the experiment, across animals.

\subsection{Strength duration curves}

The nerve excitability was studied using strength duration curves. The stimulation threshold for a given fiber type or component was defined as the current at which the CNAP amplitude for a given fiber type is above $5 \mu \mathrm{V}$. The strength duration curves were constructed using the Lapicque formula:

$$
I_{\mathrm{th}}=\frac{I_{\mathrm{rh}}}{1-e^{\tau_{\mathrm{pw}} / \tau_{m}}},
$$

where $I_{\mathrm{th}}$ is the threshold current, $I_{\mathrm{rh}}$ is the rheobase current, $\tau_{\mathrm{pw}}$ is the stimulation pulsewidth and $\tau_{m}$ is the nerve fiber membrane time constant. 


\subsection{Statistical analysis}

All numerical data were analyzed by either using paired $t$-test or Student's $t$-test.

\section{Results}

Typical results of our CNAP measurements are shown in Fig. 3, presented for three pulsewidths: $0.1,0.2$ and $0.5 \mathrm{~ms}$ (measurements for $1 \mathrm{~ms}$ and $5 \mathrm{~ms}$ not shown) and 16 stimulation current levels. The CNAPs are divided into different velocity regions as demonstrated in Fig. 22 in order to analyze contribution from different fiber types.

\subsection{Fiber classification}

The analysis of CNAP waveform and its velocity profile indicates the presence of different fiber types, their respective fiber velocities and the effect on the conduction velocities due to injections.

In Fig. 4(a), example results are shown for identifying the CNAP peaks and then subsequent generation of a raster plot based on these peaks, Fig. (4b). These plots give a clear indication of the fiber types. The position of the boundaries between different fiber types (blue dashed lines) is established on the basis of fiber velocity calculations. Finally, Fig. $4(\mathrm{c})$ presents an FEvPD graph generated from the raster plot.

\subsubsection{Fiber velocity}

After the identification of various fiber types, the range of velocities for each individual fiber type and the mean velocities are reported in Table [1] The mean velocities of these different fiber types, shown in Fig. A.4(a), were significantly different

Table 1. Classification of SubDiaGVN based on velocity and percentage of fiber events.

\begin{tabular}{lccc}
\hline Fiber type & $\begin{array}{c}\text { Velocity } \\
\text { range } \\
\left(\mathrm{ms}^{-1}\right)\end{array}$ & $\begin{array}{c}\text { Mean } \\
\text { velocity } \\
\left(\mathrm{ms}^{-1}\right)\end{array}$ & $\begin{array}{c}\text { Percentage } \\
\text { events }\end{array}$ \\
\hline $\mathrm{A} \delta$ & $>4.5$ & 5.1 & 1.8 \\
$\mathrm{~B}$ & $4.5-2.0$ & 2.47 & 38.7 \\
$\mathrm{C} 1$ & $2.0-1.2$ & 1.35 & 31.7 \\
$\mathrm{C} 2$ & $1.2-0.77$ & 0.97 & 21.6 \\
$\mathrm{C} 3$ & $0.77-0.57$ & 0.62 & 5.3 \\
$\mathrm{C} 4$ & $<0.57$ & 0.45 & 0.81 \\
\hline
\end{tabular}

from each other. We also investigated whether the fiber velocities were affected by different stimulation pulsewidths for different fiber types, and found no difference between them as shown in Fig. A.4(a). We note that for $\mathrm{C} 1$ fibers, a small decrease can be observed, but with no statistical significance $(p=$ $0.08)$.

Also, with increase in stimulation current, the $\mathrm{C} 1$ fiber velocity distribution will shift towards lower velocity due to recruitment of slower fibers. This can be observed on the color map, particularly, for pulsewidths $0.2 \mathrm{~ms}$ and $0.5 \mathrm{~ms}$, in Fig. 3

The mean fiber velocities also did not shift significantly under different physiological conditions, Fig. A.4(b). Since fiber velocity does not change significantly under different stimulation and physiological conditions, it can be used as a parameter to determine fiber damage due to implantation in an implanted solution or at the end of in vivo experiments.

\subsection{2. $\quad$ Stimulation thresholds}

The mean stimulation threshold was determined for different animals and SD curves were calculated. The rheobase current for $\mathrm{A}, \mathrm{B}$ and $\mathrm{C} 1$ fibers are $0.28( \pm 0.13), 0.46( \pm 0.26) \mathrm{mA}$ and $0.66( \pm 0.21) \mathrm{mA}$, respectively, obtained by fitting the results to Eq. (2). As observed in the previous papers, the stimulation thresholds for A fibers are the lowest followed by B and C fibers!

\subsection{Effect of CCK}

Next, we investigate how various physiological conditions affect the fiber velocity, stimulation threshold and CNAP amplitude and power. The four different conditions are described in the methods. The fiber velocities were not observed to be affected due to different injections as shown in Fig. A.4. No statistical differences exist between mean fiber velocity preand post-injections.

\subsubsection{Stimulation thresholds and CNAP amplitude}

Figure 5 shows an example of the CNAP waveforms on the SubDiaGVN for four different physiological conditions and same stimulation currents and pulsewidth. Firstly, we can note that even the 


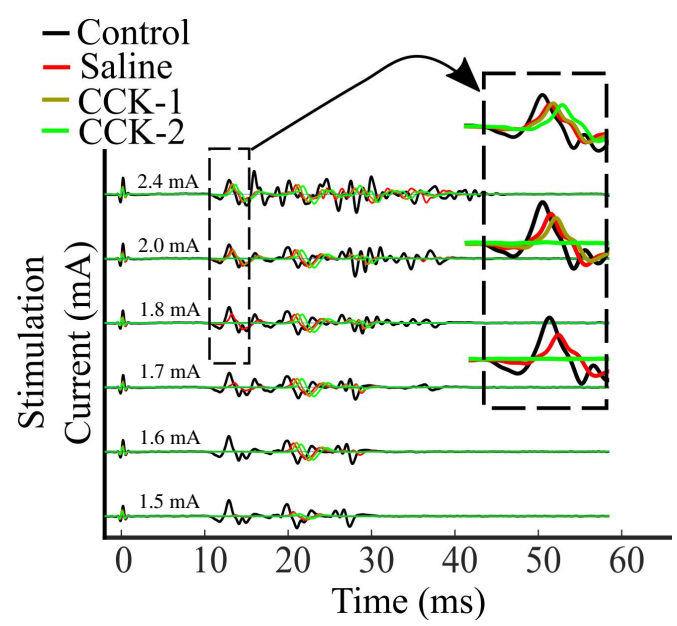

Fig. 5. An example of variations in stimulation thresholds and CNAP amplitudes under different conditions (Control, Saline, CCK1, CCK2), for $0.1 \mathrm{~ms}$ pulsewidth and $2 \mathrm{~Hz}$ stimulation frequency.

injection of saline has created a change in the CNAP response: the stimulation threshold increased and the amplitude was reduced for the stimulation threshold current. However, additional variations in respect to the Saline condition are observed for CCK injections.

Systematic investigations of the CCK effects across different fiber types and animals are shown in Fig. 6. The left column panels show the results within first 2 min after injections (i.e. when CCK is active) and the right-hand column panels show the results starting at 4 min after injection, when CCK is largely absent because its life-time is approximately 2-3 min.1925131 We find that for myelinated nerve fibers such as A and B, CNAP components, as shown in Fig. [6(a), exhibited increased stimulation thresholds. A pairwise $t$-test indicates statistically significant differences between stimulation thresholds for Saline-CCK1 $(p=0.02)$ and Saline-CCK2 $(p=$ 0.007).

On the contrary, the results for unmyelinated Cfibers shown in Fig. 7 demonstrate a trend of slightly lower stimulation thresholds when CCK is active, however, the result was not statistically significant enough $(p=0.3)$.

Additionally, we have investigated the number of CNAP peaks for all conditions across different animals, results are shown in Fig. A.5 The figure shows fiber events normalized to the total fiber events in the CNAP waveform. The normalized fiber event count is significantly reduced under the presence of CCK2

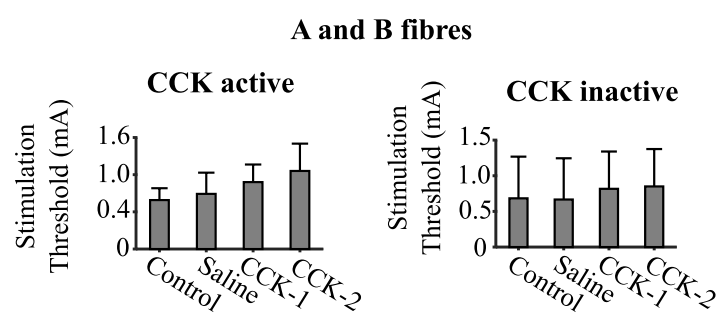

(a)

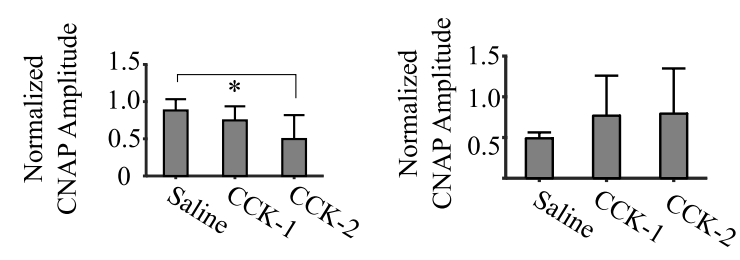

(b)

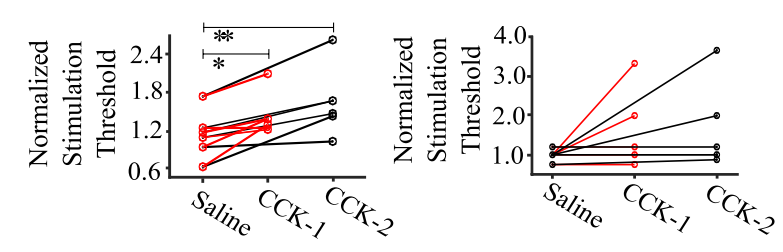

(c)

Fig. 6. Effect of injections on CNAPs from myelinated ( $\mathrm{a}$ and $\mathrm{b}$ ) fibers is shown here. A and B fibers exhibit: (a) higher stimulation threshold currents and (b) CNAP amplitudes normalized to CNAP amplitudes observed during Control. Left panels show results up to $90 \mathrm{~s}$ after injections (in the case of CCK injections, CCK will be still active) and right panels are measurements of $250 \mathrm{~s}$ after injections (when CCK becomes inactive). CCK has a relatively short half-life in the circulation, approxi-

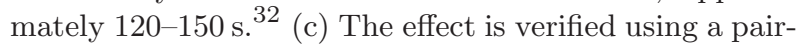
wise $t$-test on normalized (to Control) stimulation threshold with $p_{\text {Saline-CCK1 }}=0.02$ and $p_{\text {Saline-CCK2 }}=0.007$.

for $\mathrm{A}$ and $\mathrm{B}(p=0.05$ for both, using the simple $t$-test). Furthermore, the B fibers show significant decrease when CCK is present compared to Saline as well $(p=0.03)$.

\section{Discussion}

This paper presents the results of a study into the electrophysiological response of SubDiaGVN, which is relevant for appetite control. This study is relevant for neurometabolic therapies, which are very promising neurotechnologies for a number of medical conditions including obesity and obesity-related risk factors, such as high cholesterol or high blood pressure. ${ }^{5.114 \mid} \mathrm{By}$ investigating the CNAP waveforms 


\section{C fibres}

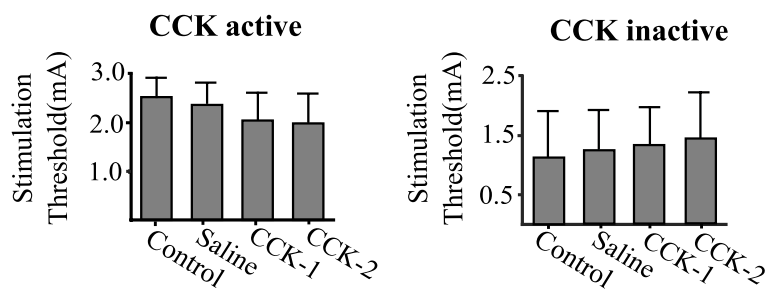

(a)
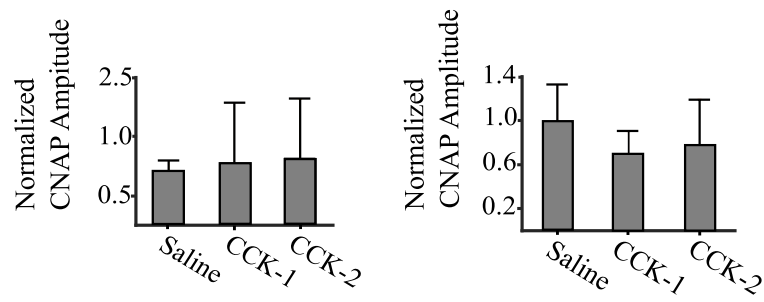

(b)

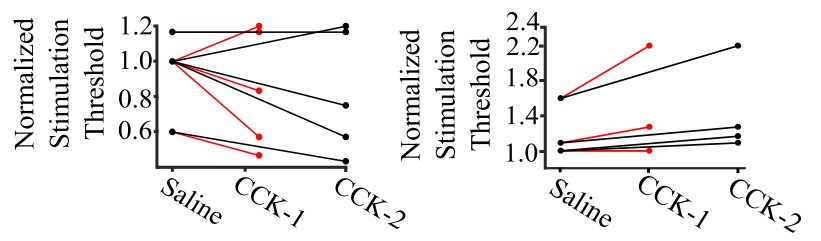

(c)

Fig. 7. Effects of the injections on CNAPs from unmyelinated (c) fibers, explanations for different panels as in Fig. 6

elicited in the SubDiaGVN, we were able to identify the fiber types present in that branch of the vagus and establish the effect of physiological stimuli, such as CCK, on the CNAPs.

The methodology described in this paper has enabled the identification of different fiber types present in the SubDiaGVN. The nerve stimulationrecording approach has been previously investigated, for example, by Tahry et al ${ }^{[33}$ in human studies of VNS for epilepsy in order to evaluate the elicited activation of the nerve, and very recently by Ward et al ${ }^{\sqrt{18}}$ to monitor the level of nerve activation. However, in both cases, the stimulation and recording sites were close to each other, thus requiring a special technique to cancel the stimulation artifact in order to prevent saturation of the neural recording amplifier and to resolve CNAP components from the artifact. It was difficult to clearly identify boundaries between CNAP components from different fiber types. In our case, the distance is $8 \mathrm{~cm}$ in the rat model. This enables an easy fiber distinction on the basis of CNAP conduction velocities. Here, the fiber classification was done in accordance with the Letter System,, 28 with previously established boundaries for rat vagus $\underline{34}$

A benefit of using CerVN as a preferred stimulation site is that it is a popular stimulation site for a range of VNS applications, such as epilepsy and depression. It enables interrogation of the state of the nerve at any branch of the vagus nerve below the neck with the same methodology and by changing only the recording location. Reported side effects of VNS at CerVN include hoarseness and paresthesia, which, however, exhibit lower long-term incidence ${ }^{[356}$ Clinical studies are used to demonstrate VNS efficacy for obesity report mild or moderate side effects such as heartburn, dyspepsia, pain, dysphagia and nausea. ${ }^{[6}$ Due to recent advances in semiconductor technology and body area networks, it is possible to incorporate this methodology in a small size implant, however, two different incisions are required and the interrogative stimulation protocol has to be optimized in order to prevent activation of offtarget pathways, especially, the heart. The stimulation parameters described in this paper did not lead to a reduction in heart rate. Also, a reason to separate the recording and stimulation sites is to improve resolution of CNAPs originating from different fiber types. A distance of $8 \mathrm{~cm}$ seems acceptable to achieve good resolution, hence in larger physiological models, such as humans, a CerVN stimulation site is not required if the goal is to characterize a specific branch such as the SubDiaGVN.

\subsection{Fiber classification}

The analysis of SubDiaGVN CNAPs demonstrates the presence of primarily A, B and C1 fibers. Other types of slow $\mathrm{C}$ fibers such as $\mathrm{C} 2-\mathrm{C} 4$ were also observed in some animals, however, not consistently. The mean fiber velocities for $\mathrm{A}, \mathrm{B}$ and $\mathrm{C}$ fibers differed significantly $\left(p_{\mathrm{A}-\mathrm{B}}=2.5 \cdot 10^{-4}, p_{\mathrm{A}-\mathrm{C}}=\right.$ $8.1 \cdot 10^{-7}$ and $\left.p_{\mathrm{B}-\mathrm{C}}=0.002\right)$ for pulsewidth of $0.1 \mathrm{~ms}$. After variation of pulsewidth, the mean fiber velocities do not change significantly. As the mean fiber velocity does not change significantly across pulsewidths and across conditions, it indicates that the nerve was functional and healthy throughout the experiment. 
Fiber distribution, based on the number of fiber events in CNAPs and hence FEvPD, appears similar to observations made by Precthl et al ${ }^{20}$ where it was demonstrated that the SubDiaGVN branch comprises of a majority of unmyelinated fibers such as $\mathrm{C}$ fibers, which carry sensory information.20

The range of stimulation currents $(0.1-3 \mathrm{~mA})$ and pulsewidths $(0.1-5 \mathrm{~ms})$ considered in this paper covers the traditional range of current and pulsewidths in use for VNS therapies. ${ }^{33135137}$ The stimulation thresholds for different fiber types are significantly different from each other. The rheobase currents for $\mathrm{A}, \mathrm{B}$ and $\mathrm{C}$ fibers observed in these experiments are $0.28( \pm 0.13), 0.46( \pm 0.26)$ and $0.68( \pm 0.21) \mathrm{mA}$. The thresholds for A and B fibers are higher than thresholds reported for CerVN in rats earlier which were in the order of $0.01,0.03$ and $0.2 \mathrm{~mA} .30$ Possible reasons could be lower percentage of $\mathrm{A}$ and $\mathrm{B}$ fibers and a higher concentration of C fibers in the SubDiaGVN branch, 20 where signals were recorded. Other possible reasons could depend on site of stimulation and type of electrodes used for stimulation.

\subsection{Effect of CCK}

To our best knowledge, this study presents the first results demonstrating the impact of CCK- 8 on CNAPs originating from different fiber types, without nerve fiber dissection. Thus, our study is a contribution to investigations of the interaction between two different appetite control pathways gut hormonehypothalamus and vagus nerve-brainstem, and to the fundamental question of interaction between the hormonal and neural systems. The effect of CCK on vagal efferents is observed on the stimulation threshold and amplitude of CNAP components originating from $\mathrm{A}$ and $\mathrm{B}$ fibers, which become less excitable. Recent work on the effect of CCK-8 on ventral gastric vagus nerve which innervates the stomach shows that intravenous CCK- 8 injection produces an inhibitory effect on efferent fibers and an opposite effect on afferent fibers using mass activity and fiber dissection 19 The inhibitory effect on efferent fibers leads to a reduced gastric tone and contractile activity, leading to a feeling of satiation. 19

The effect of CCK on normalized stimulation thresholds of CNAPs, observed in this study, can be detected, hence providing a potential biomarker to answer when to stimulate. However, no statistically significant difference was observed between two doses of CCK used in this study, as shown in Fig. [6] to allow stimulation dose determination as well. The dose regulation can be achieved by nerve characterization described here and selecting stimulation parameters based on this, making the stimulation dosage patient-specific. As therapeutic peripheral nerve stimulation requires excitation or blockage of all or specific fiber type activity, stimulation parameters can be intelligently chosen or changed based on this.

We observed certain differences in CNAP waveforms when injecting Saline only. This could be due to increased blood pressure caused by injection of additional fluid volume into the vascular system and consequent reaction by the vagus nerve to that change. However, the changes observed due to CCK are in addition to Saline and statistically significant.

Furthermore, we observed occasional variations of the recorded artifact (created by the electrical stimulation) for various injections. However, they occur because of input stage filtering (the band pass filter acts effectively as a differentiator) and insufficient sampling rate $(20 \mathrm{kHz})$ to observe fast voltage transitions. The conclusion is that the stimulus artifact does not show any systematic trends (e.g. attenuation), and it does not reflect change in stimulation thresholds for different conditions, see Fig. B.1.

With current advances in semiconductor technology, it is possible to integrate processing algorithms into implantable devices.2] The AspireSR (Cyberonics) system is a commercially available closed loop VNS implant to treat epilepsy, in which the question when to stimulate is answered by processing cardiac signals to detect seizure-related ictal tachycardia .111 Recently, we also demonstrated how extracellular $\mathrm{pH}$ changes due to the SubDiaGVN activity can be utilized in the closed loop paradigm $[38$ Furthermore, we are currently investigating how other chemical signals of neural activity, such as potassium ions concentration, could be used for this purpose. The advantage of using chemical signals would be better signal to noise ratio in comparison to detecting, say the mass activity changes. 
In this study, order effects were not considered. This is due to our observation that the nerve integrity has been preserved and the excitability levels have not shown statistically significant changes as confirmed in our stimulation protocol safety study (see Fig. B.2). Furthermore, there was no overall change in vitals such as heart rate, throughout the experiment. However, for future studies, randomized order of physiological conditions can be considered.

\section{Conclusion}

Neurometabolic therapies are an interesting and recently discovered area of application for neuromodulation devices. However, in such therapies, it is crucial to understand the impact of physiological metabolic control systems on neural stimulation and vice versa, to deliver therapeutic stimulation dosage in an efficient manner. In this study, an effort has been made to understand the composition of the SubDiaGVN and the impact of appetite reducing gut hormone CCK on its signaling. The motivation was to use the results for future development of a closed loop VNS implant which can help link the two independent appetite control pathways, and through stimulation amplify the appetite reducing effect of gut hormone CCK. Existing closed loop systems for obesity, such as the Diamond ${ }^{\mathrm{TM}}$ (Metacure), initiate stimulation on conduit of food in the stomach. It is however unclear how the stimulation dose is controlled. In comparison, the methodology proposed in the paper could enable when and how much to stimulate and possibly provide greater therapeutic efficiency by seeking to link metabolic and neural control pathways. Here, we have shown that CCK reduces activity of $\mathrm{A}$ and $\mathrm{B}$ fibers. The inhibitory effect of the gut hormone, when detected, can be used as a trigger to initiate stimulation. Furthermore, the nerve classification protocol and CNAPs can be used as a means to identify the impact of stimulation on the state of the nerve, such as percentage activation or inhibition of specific nerve fiber types.

Further studies will lead us to better understanding of the physiology of the gastric branch of the vagus nerve and its activity due to other physiological conditions. Ultimately, we might be able to understand the coding of the nerve in the process of appetite regulation to design better neurometabolic therapies for obesity management, appetite control and weight loss.

\section{Acknowledgments}

This work was supported by the European Research Council (Synergy Grant No. 319818, i2MOVE) and UK EPSRC grant EP/N002474/1. We would like to thank Dr. Claudio Zuliani, Dr. Sivylla Paraskevopoulou and Mr. Nishanth Kulasekeram for technical assitance, Dr. Ioannis Christakis and Dr. Agnieska Falinska for surgical input and Dr. Miguel Angel Ibánez for biostatistical advice.

\section{Appendix A}

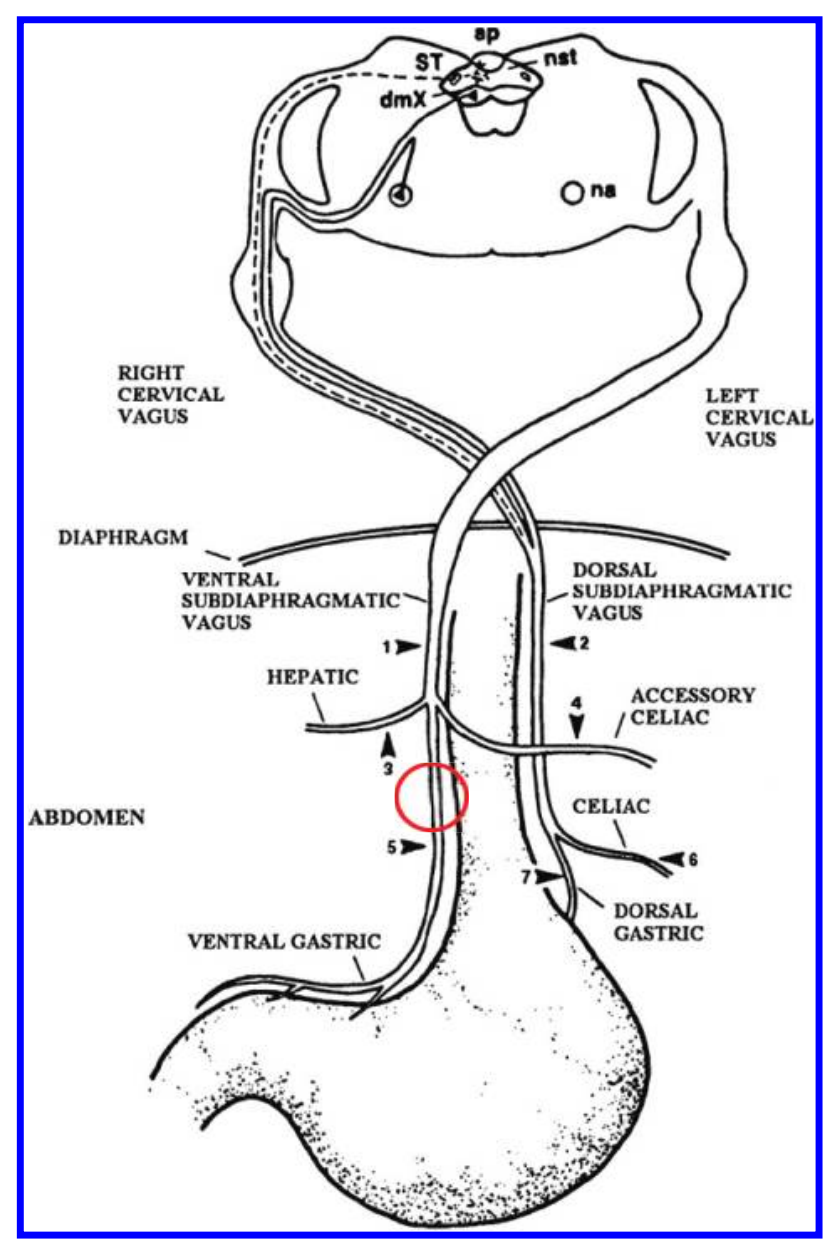

Fig. A.1. (Color online) Left ventral subDiaGVN in a rat (red circle), from which CNAPs were recorded during the experiment.

Source: Dixon et al., Am. J. Physiol. (2000). 


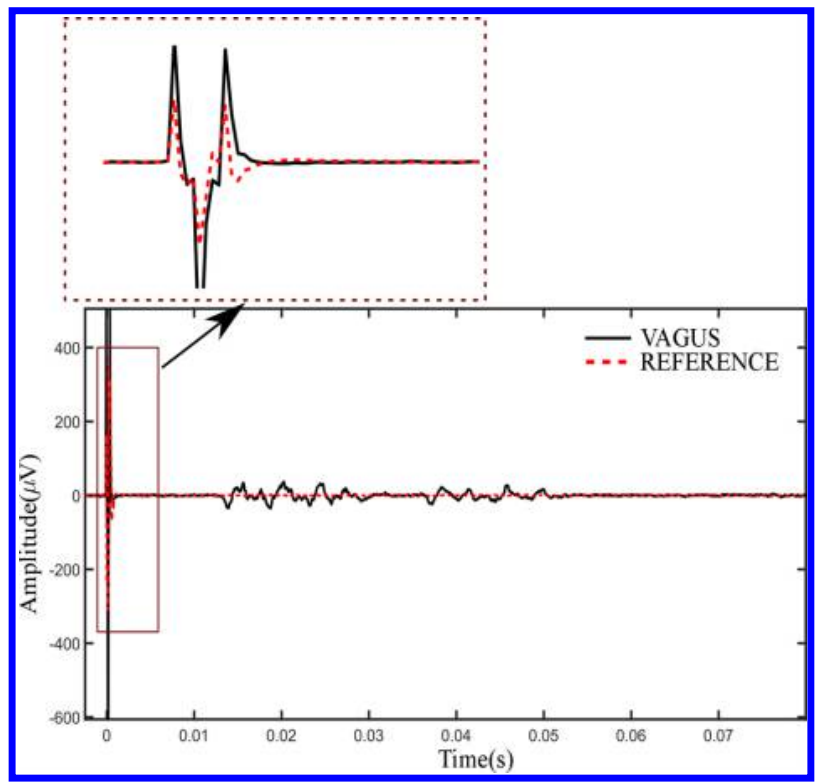

Fig. A.2. The vagus recording channel is overlaid with the subdiaphragmatic reference channel, which is used for comparison to differentiate CNAPs from interferences (due to movement, EMG, etc.). The inlet shows stimulation artifact alignment for the two channels. In this example, the CNAPs were elicited at the following stimulation parameters: stimulation current $2 \mathrm{~mA}$, pulse width $0.2 \mathrm{~ms}$, and stimulus frequency $2 \mathrm{~Hz}$.

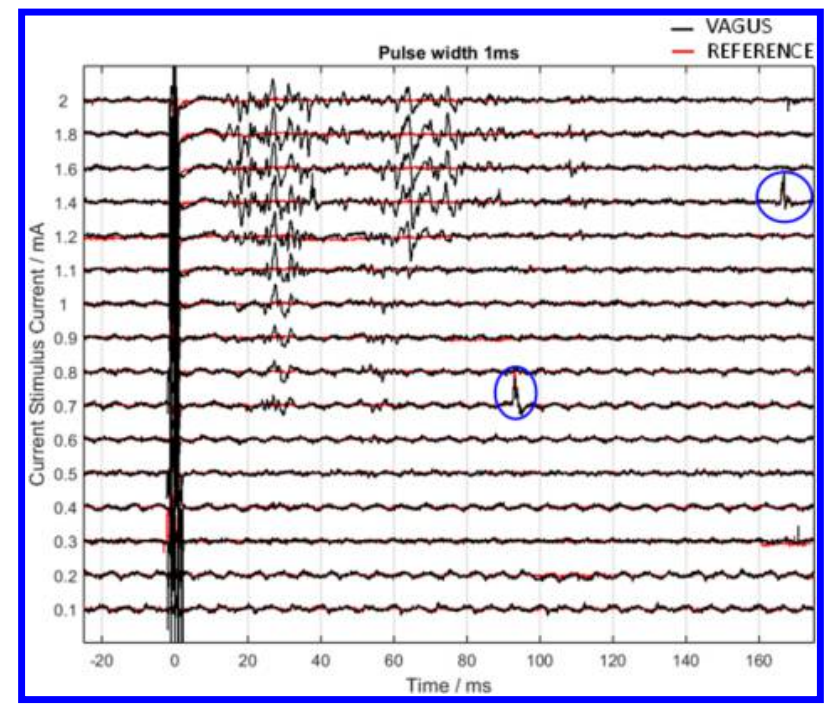

Fig. A.3. (Color online) The CNAPs elicited, during an experiment, for 16 different currents at $1 \mathrm{~ms}$ pulsewidth and $2 \mathrm{~Hz}$ frequency are shown here. The components (encircled in blue) are artifacts common to both vagus and subdiaphragmatic reference channel. These peaks/components are ignored during analysis.

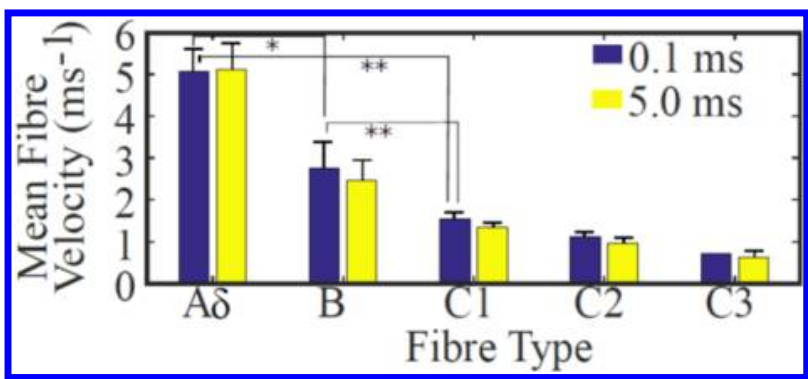

(a)

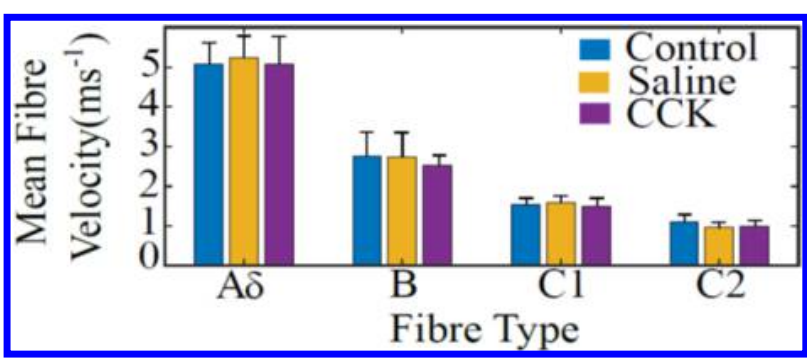

(b)

Fig. A.4. Mean fiber velocities and standard deviation for each fiber type and (a) two pulsewidths and (b) different physiological conditions. Statistically significant differences are shown with $* *(\mathrm{p}<0: 01)$ and $*(p<0: 05), n=7$ animals. 

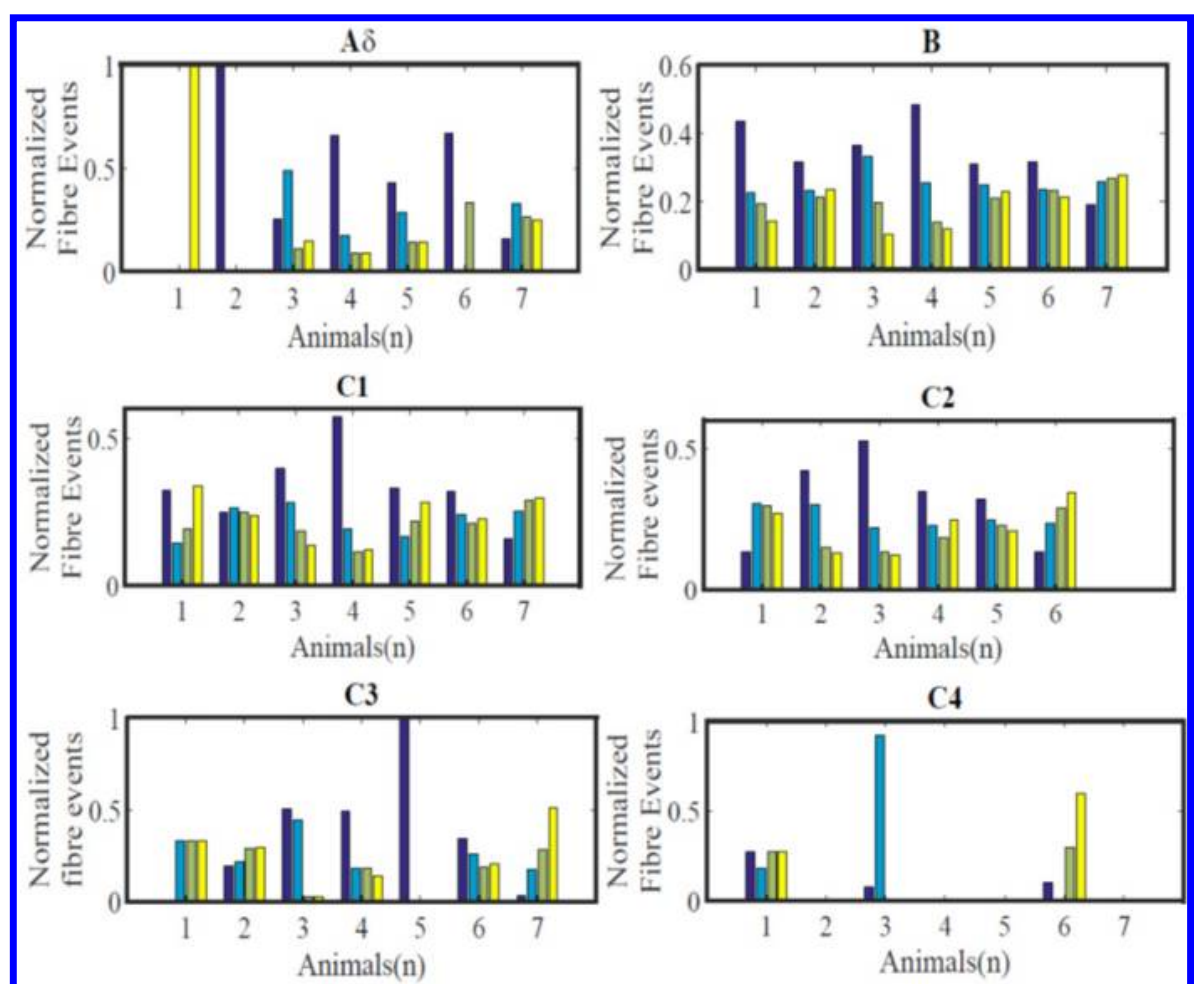

- Control Saline $1 \mathrm{CCK}-100 \mathrm{pmol}$ - $\mathrm{CCK}-1000 \mathrm{pmol}$

Fig. A.5. Variation in the number of peaks for different animals under different conditions: Control, Saline, CCK-low, CCK-high dose (and measured during the period when CCK is active). 


\section{Appendix B}

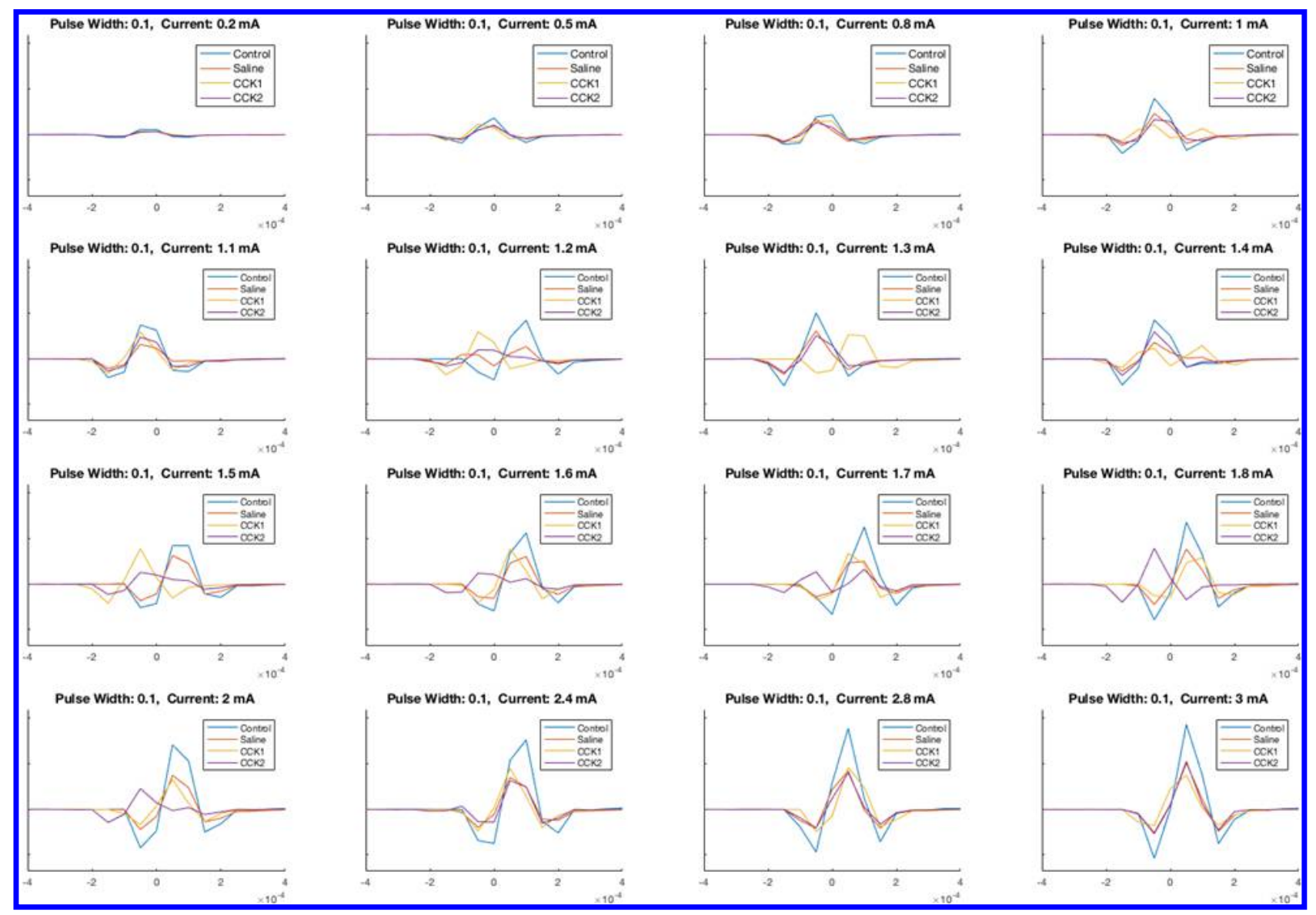

(a)

Fig. B.1. Stimulus artifact under different conditions (Control, Saline, CCK1, CCK2) for $0.1 \mathrm{~ms}$ pulsewidth and 16 stimulation currents: (a) Experiment shown in Fig. [7 (b) Another experiment. Artifact variability is due to low sampling rate (20 per $\mathrm{ms}$ ) and band pass filtering $200 \mathrm{~Hz}-7.2 \mathrm{kHz}$. 


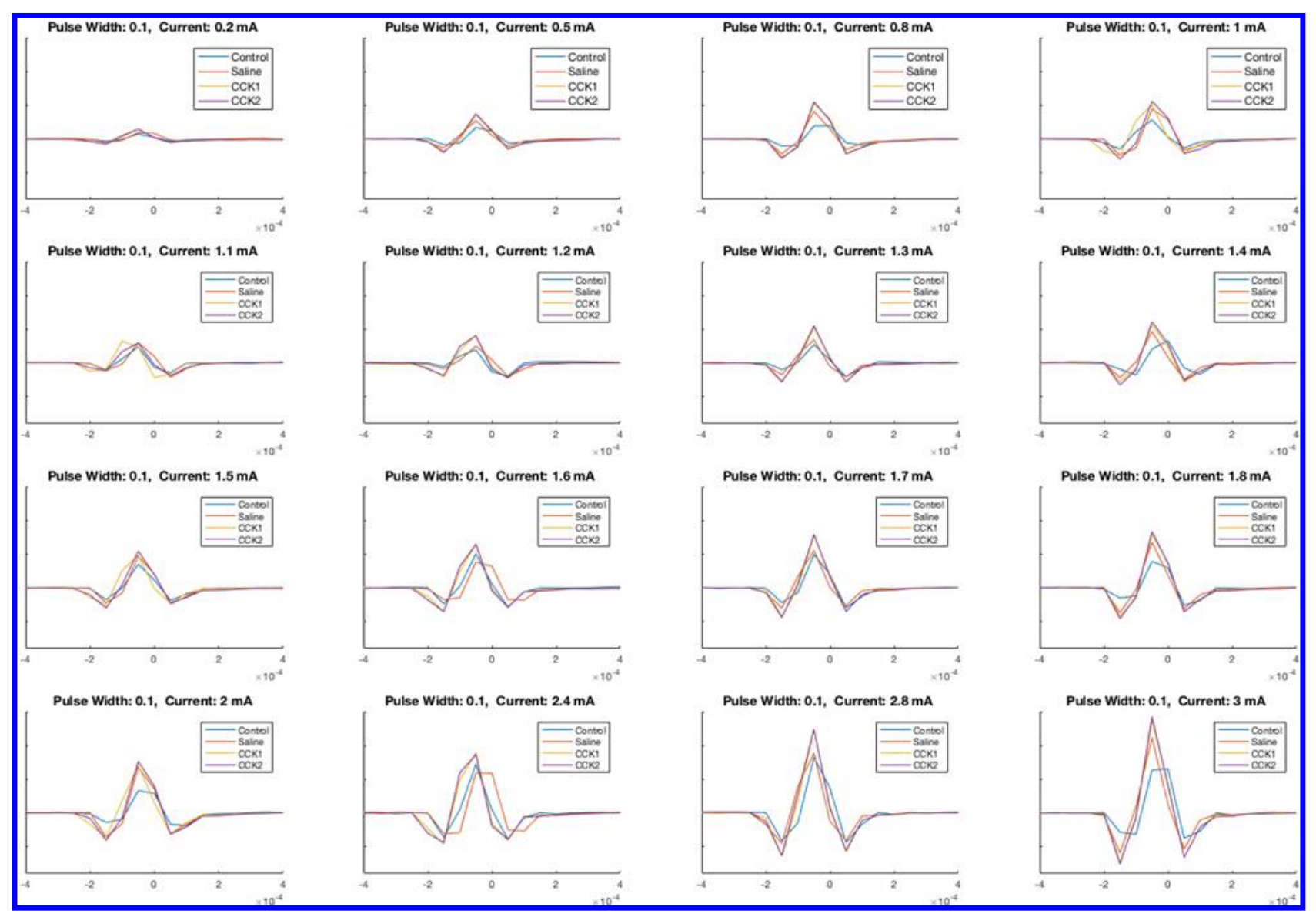

(b)

Fig. B.1. (Continued) 


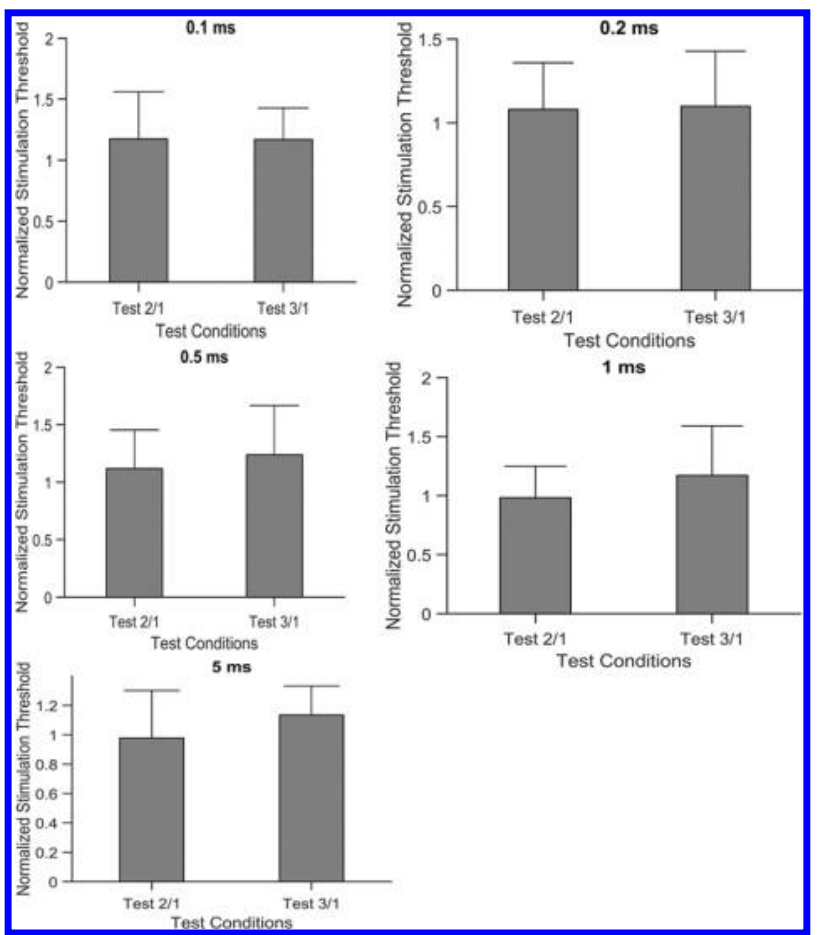

Fig. B.2. The stimulation protocol was tested for safety in $n=8$ animals. The complete stimulation protocol, five pulsewidths shown in five panels above, and 16 different currents $(2 \mathrm{~Hz}$ stimulation frequency, $10 \times$ repeated each pulsewidth/stimulation current combination and averaged), was repeated three times (Test 1, Test 2, Test 3, resting time between Tests $10 \mathrm{~min}$ ) in a similar manner as described in the paper, however, without any injections. The stimulation thresholds for A or B fibers in Test 2 and Test 3 were normalized to the stimulation thresholds of Test 1 , and the mean and standard error are shown. The normalized stimulation thresholds were not significantly different from each other. A similar result was obtained for $\mathrm{C}$ fibers. Hence, we conclude that the stimulation protocols used in our experiments do not affect the nerve excitability.

\section{References}

1. B. Bonaz, C. Picq, V. Sinniger, J. F. Mayol and D. Clarençon, Vagus nerve stimulation: From epilepsy to the cholinergic anti-inflammatory pathway, Neurogastroenterol. Moti. 25 (2013) 208-221.

2. S. Luan, I. Williams, K. Nikolic and G. Timothy, Neuromodulation: Present and emerging methods, Front. Neuroeng. 7(27) (2014) 1-9.

-3. L. Mollet, R. Raedt, J. Delbeke, R. El Tahry, A. Grimonprez, I. Dauwe, V. De Herdt, A. Meurs, W. Wadman, P. Boon and K. Vonck, Electrophysiological responses from vagus nerve stimulation in rats, Int. J. of Neural Syst. 23(06) (2013) 1350027.

-4. S. Wostyn, W. Staljanssens, L. De Taeye, G. Strobbe, S. Gadeyne, D. Van Roost, R. Raedt, K.
Vonck and P. van Mierlo, EEG derived brain activity reflects treatment response from vagus nerve stimulation in patients with epilepsy, Int. J. Neural Syst. 27(04) (2017) 1650048.

-5. S. Ikramuddin, R. P. Blackstone, A. Brancatisano, J. Toouli, S. N. Shah, B. M. Wolfe, K. Fujioka, J. W. Maher, J. Swain, F. G. Que, J. M. Morton, D. B. Leslie, R. Brancatisano, L. Kow, R. W. O'Rourke, C. Deveney, M. Takata, C. J. Miller, M. B. Knudson, K. S. Tweden, S. A. Shikora, M. G. Sarr and C. J. Billington, Effect of reversible intermittent intraabdominal vagal nerve blockade on morbid obesity: The ReCharge randomized clinical trial, Jama 312 (2014) 915-922.

-6. D. Guiraud, D. Andreu, S. Bonnet, G. Carrault, P. Couderc, A. Hagège, C. Henry, A. Hernandez, N. Karam, V. Le Rolle, P. Mabo, P. Maciejasz, C.-H. Malbert, E. Marijon, S. Maubert, C. Picq, O. Rossel and J.-L. Bonnet, Vagus nerve stimulation: State of the art of stimulation and recording strategies to address autonomic function neuromodulation, $J$. Neural Eng. 13(4) (2016) 041002.

-7. H. R. Berthoud, The vagus nerve, food intake and obesity, Regul. Pept. 149 (2008) 15-25.

-8. R. C. Ritter, Gastrointestinal mechanisms of satiation for food, Physiol. Behav. 81(2) (2004) 249-273.

9. M. G. Sarr, C. J. Billington, R. Brancatisano, A. Brancatisano, J. Toouli, L. Kow, N. T. Nguyen, R. Blackstone, J. W. Maher, S. Shikora, D. N. Reeds, J. C. Eagon, B. M. Wolfe, R. W. O'Rourke, K. Fujioka, M. Takata, J. M. Swain, J. M. Morton, S. Ikramuddin, M. Schweitzer, B. Chand and R. Rosenthal, The EMPOWER study: Randomized, prospective, double-blind, multicenter trial of vagal blockade to induce weight loss in morbid obesity, Obes. Surg. 22 (2012) 1771-1782.

10. G. Lartigue, Role of the vagus nerve in the development and treatment of diet-induced obesity, J. Physiol. 594(20) (2016) 5791-5815.

11. F. T. Sun and M. J. Morrell, Closed-loop Neurostimulation: The clinical experience, Neurotherapeutics 11(3) (2014) 553-563.

12. L. Busetto, A. J. Torres, S. Morales-Conde, I. Alarcón del Agua, C. Moretto, P. Fierabracci, G. Rovera, G. Segato, M. A. Rubio and F. Favretti, Impact of the feedback provided by a gastric electrical stimulation system on eating behavior and physical activity levels, Obesity 25(3) (2017) 514-521.

13. T. Horbach, A. Thalheimer, F. Seyfried, F. Eschenbacher, P. Schuhmann and G. Meyer, abiliti $(\mathbb{R}$ closed-loop gastric electrical stimulation system for treatment of obesity: Clinical results with a 27month follow-up, Obes. Surg. 25(10) (2015) 17791787.

14. H. Johannessen, D. Revesz, Y. Kodama, N. Cassie, K. Skibicka, P. Barrett, S. Dickson, J. Holst, J. Rehfeld, G. van der Plasse, R. Adan, B. Kulseng, E. Ben-Menachem, C. M. Zhao and D. Chen, Vagal 
blocking for obesity control: A possible mechanismof-action, Obes. Surg. 27 (2016) 1-9.

15. G. J. Dockray, Cholecystokinin and gut-brain signalling, Regul. Pept. 155(1) (2009) 6-10.

-16. M. Covasa and R. C. Ritter, Reduced sensitivity to the satiation effect of intestinal oleate in rats adapted to high-fat diet, Am. J. Physiol. Regul. Integr. Comp. Physiol. 277(1) (1999) R279R285.

17. A. H. Sam, R. C. Troke, T. M. Tan and G. A. Bewick, The role of the gut/brain axis in modulating food intake, Neuropharmacology 63(1) (2012) 46-56.

18. M. P. Ward, K. Y. Qing, K. J. Otto, R. M. Worth, S. W. John and P. P. Irazoqui, A flexible platform for biofeedback-driven control and personalization of electrical nerve stimulation therapy, IEEE Trans. Neural Syst. Rehabil. Eng. 23(3) (2015) 475-484.

19. S. Okano-Matsumoto, J. A. McRoberts, Y. Taché and D. W. Adelson, Electrophysiological evidence for distinct vagal pathways mediating CCK-evoked motor effects in the proximal versus distal stomach, J. Physiol. 589(2) (2011) 371-393.

20. J. C. Prechtl and T. L. Powley, The fiber composition of the abdominal vagus of the rat, Anat. Embryol. 181(2) (1990) 101-115.

21. J. Kimura, Electrodiagnosis in Diseases of Nerve and Muscle: Principles and Practice (Oxford university press, Oxford, 2001).

22. C. Maggi and A. Meli, Suitability of urethane anesthesia for physiopharmacological investigations in various systems part 1: General considerations, Cell. Mol. Life Sci. 42(2) (1986) 109-114.

-23. J. R. Potas, N. G. De Castro, T. Maddess and M. N. De Souza, Waveform similarity analysis: A simple template comparing approach for detecting and quantifying noisy evoked compound action potentials, PLoS One 10(9) (2015) 1-18.

24. M. Sahin, M. A. Haxhiu, D. M. Durand and I. A. Dreshaj, Spiral nerve cuff electrode for recordings of respiratory output, J. Appl. Physiol. 87(2197-2206) (1999) 317-3122.

25. V. Bucinskaite, M. Kurosawa and T. Lundeberg, Exogenous cholecystokinin-8 reduces vagal efferent nerve activity in rats through $\mathrm{CCK}(\mathrm{A})$ receptors, $B r$. J. Pharmacol. 129(8) (2000) 1649-54.

-26. V. Bucinskaite, T. Tolessa, J. Pedersen, B. Rydqvist, L. Zerihun, J. J. Holst and P. M. Hellström, Receptor-mediated activation of gastric vagal afferents by glucagon-like peptide-1 in the rat, Neurogastroenterol. Motil. 21 (2009) 978.
27. R. R. Harrison, A versatile integrated circuit for the acquisition of biopotentials, in Proc. Custom Integrated Circuits Conf. (IEEE, San Jose, USA, 2008), pp. $115-122$.

28. H. S. Gasser, The classification of nerve fibers., Ohio J. Sci. 41 (1941) 145-159.

29. L. N. S. Andreasen and J. J. Struijk, Signal strength versus cuff length in nerve cuff electrode recordings, IEEE Trans. Biomed. Eng. 49(9) (2002) 1045-1050.

30. D. M. Woodbury and J. W. Woodbury, Effects of vagal stimulation on experimentally induced seizures in rats, Epilepsia 31(s2) (1990) S7-S19.

-31. M. Kurosawa, K. Uvnäs-Moberg, K. Miyasaka and T. Lundeberg, Interleukin-1 increases activity of the gastric vagal afferent nerve partly via stimulation of type A CCK receptor in anesthetized rats, J. Auton. Nerv. Syst. 62(1-2) (1997) 72-78.

-32. K. P. Skibicka and S. L. Dickson, Enteroendocrine hormones central effects on behavior, Curr. Opin. Pharmacol. 13(6) (2013) 977-982.

-33. R. El Tahry, R. Raedt, L. Mollet, V. De Herdt, T. Wyckhuys, T. Wyckuys, A. Van Dycke, A. Meurs, F. Dewaele, D. Van Roost, P. Doguet, J. Delbeke, W. Wadman, K. Vonck and P. Boon, A novel implantable vagus nerve stimulation system (ADNS300) for combined stimulation and recording of the vagus nerve: Pilot trial at Ghent University Hospital, Epilepsy Res. 92 (2010) 231-239.

-34. Y. Yamazaki, T. Karakida and S. Homma, Conduction velocity of motor nerve and cervical sympathetic and vagus nerve in streptozotocin diabetic rats, Neurosci. Lett. 113(1) (1990) 29-33.

-35. A. P. Amar, M. L. Levy, C. Y. Liu and M. L. J. Apuzzo, Vagus nerve stimulation, Proc. IEEE 96(7) (2008) 1142-1151.

36. P. Boon, K. Vonck, J. D. Reuck and J. Caemaert, Vagus nerve stimulation for refractory epilepsy, Seizure 10(6) (2001) 448-455.

37. G. M. De Ferrari, H. J. G. M. Crijns, M. Borggrefe, G. Milasinovic, J. Smid, M. Zabel, A. Gavazzi, A. Sanzo, R. Dennert, J. Kuschyk, S. Raspopovic, H. Klein, K. Swedberg and P. J. Schwartz, Chronic vagus nerve stimulation: A new and promising therapeutic approach for chronic heart failure, Eur. Heart J. 32 (2011) 847-855.

38. S. Cork, A. Eftekhar, K. B. Mirza, C. Zuliani, K. Nikolic, J. Gardiner, S. Bloom and C. Toumazou, Extracellular ph monitoring for use in closed-loop vagus nerve stimulation, J. Neural Eng. 15(1) (2018) 016001. 